\title{
Spontaneous Symmetry Breaking in Local Gauge Quantum Field Theory; the Higgs Mechanism
}

\author{
F. Strocchi $\star \star \star$ \\ Joseph Henry Laboratories of Physics, Princeton University, Princeton, New Jersey 08540, USA
}

\begin{abstract}
Spontaneous symmetry breakings in indefinite metric quantum field theories are analyzed and a generalization of the Goldstone theorem is proved. The case of local gauge quantum field theories is discussed in detail and a characterization is given of the occurrence of the Higgs mechanism versus the Goldstone mechanism. The Higgs phenomenon is explained on general grounds without the introduction of the so-called Higgs fields. The basic property is the relation between the local internal symmetry group and the local group of gauge transformations of the second kind. Spontaneous symmetry breaking of $c$ number gauge transformations of the second kind is shown to always occur if there are charged local fields. The implications about the absence of mass gap in the Wightman functions and the occurrence of massless particles associated with the unbroken generators in the Higgs phenomenon are discussed.
\end{abstract}

\section{Introduction}

The discovery [1] that local internal symmetries in QFT (i.e. symmetries of the equations of motion or of the Hamiltonian) may fail to give rise to global symmetries of the theory because of vacuum instability suggested a simple powerful mechanism to understand symmetry breaking in elementary particle physics. Unfortunately, it was soon realized [2] and proved [3] quite generally in the framework of axiomatic (positive metric) QFT that such a mechanism requires the existence of massless particles (Goldstone bosons) with the quantum numbers of the generator of the local internal symmetry and there does not seem to be any candidate for such particles. A genuine Goldstone mechanism is therefore excluded from elementary particle physics. Actually, the only massless boson existing in nature is the photon and it is associated with an unbroken internal symmetry (gauge transformations of the first kind).

It was later shown [4] that a spontaneous symmetry breaking may occur without implying the existence of massless particles provided one is dealing with gauge field theories (Higgs phenomenon). This is not a counterexample to the

* Supported in part by NSF Grant MPS 74-22844

$\star \star$ On leave from Scuola Normale Superiore INFN, I-56100 Pisa, Italy 
general theorem [3] since in gauge field theories not all the standard axioms of positive metric formalism [5] can be satisfied, and in particular one cannot have locality covariance and positivity at the same time [6].

The occurrence of the Higgs phenomenon has been discussed in explicit Lagrangian models but it appears that a general treatment or a general theorem is lacking. Actually given a gauge field theory it is not clear under which conditions a spontaneous symmetry breaking will occur following the Higgs mechanism rather than the Goldstone mechanism, both being allowed in gauge field theory. More specifically, one may ask when the "original" massless vector field acquires a mass by "eating the Goldstone boson" [7]. Examples are in fact known in which the Higgs mechanism is only partially realized and the vector fields remain massless [8]. All this may explain why one talks of the Higgs phenomenon and not of Higgs theorem as in the case of Goldstone mechanism.

Another very interesting feature which usually accompanies the Higgs phenomenon is that the unbroken generators have massless particles associated to them, a feature which seems to be exactly realized in nature.

All this indicates

i) that spontaneous symmetry breaking may be relevant to elementary particle physics only if one deals with gauge field theory (i.e. through the Higgs mechanism),

ii) that it is very important to know under what conditions the Higgs mechanism will act, what are the characteristic features accompanying it, and in particular whether the massless particles associated to the unbroken generators are a general fact or not.

Since there are strong indications [9] (asymptotic freedom, unified theory of weak and electromagnetic interactions, quark confinement, etc.) that gauge field theories may be the only kind of QFT relevant to elementary particle physics, it seems worthwhile to have a general discussion of the Higgs phenomenon in the framework of general local QFT in order to answer the above questions. This constitutes the main result of the present paper. In particular we will provide a characterization and an explanation of the Higgs phenomenon on very general grounds without the introduction of the so-called Higgs fields.

Most of the Lagrangian field theory discussions of the Higgs phenomenon have been carried out in the unitary or radiation gauge in which neither locality nor covariance hold. It is difficult to give a general treatment of this phenomenon in the style of axiomatic field theory in such gauges, because almost all the general features of Wightman field theory are lost. We will therefore deal with a local and covariant gauge where, as we will see, it is much easier to understand the basic features of Higgs mechanism. This shows that, just as for the Goldstone theorem, the framework of Wightman field theory proves to be natural and in fact allows a simple characterization of the Higgs phenomenon.

The crucial point is to find an axiomatic field theory translation of the properties of Lagrangian field theory models which exhibit the Higgs phenomenon, such as the existence of a vector field coupling, etc. As we will see, a consequence of such a characterization is a strict connection between the Higgs phenomenon and the failure of the cluster property, a feature which has attracted much attention in relation with charge screening and quark confinement [10] after its discovery in the Schwinger model [11]. 
Quite independently of the above physical motivations the discussion of spontaneously broken symmetries in gauge field theories provided by the present paper appears as a necessary step in the extension of the results of standard (positive metric) Wightman field theory to QFT's with indefinite metric.

\section{Local and Covariant Gauge Quantum Field Theory}

In order to avoid any question of semantics and to allow an unambiguous discussion, it is necessary to spell out what we mean by a local and covariant GQFT. The following properties can be regarded as basic properties or axioms for GQFT's. Essentially, one has to give a precise meaning to the key words: local field, covariance, gauge transformations.

By a local and covariant gauge quantum field theory we mean a quantum theory satisfying the following properties.

A. Local Fields. It is defined in terms of a set of fields $\phi_{\alpha}, \alpha=1, \ldots n$, i.e. operator valued (tempered) distributions in a Hilbert space $\mathscr{H}$, with scalar product denoted by $(\cdot, \cdot)$, having a common dense domain $D$. The fields $\phi_{\alpha}$ are local, i.e. they satisfy local commutativity, and the polynomial $*$ algebra generated by the smeared fields $\phi_{\alpha}(f)$ will be denoted by $\mathscr{F}$.

Physically interesting quantities like transition amplitudes, vacuum expectation values or Green's functions, etc. are computed in terms of a bounded, hermitean, non-degenerate ${ }^{1}$ sesquilinear form $\langle\cdot, \cdot\rangle=(\cdot, \eta \cdot)$ and $\eta$ is called the metric operator $^{2}$.

B. Covariance. There is a weakly continuous representation $U(a, \Lambda)$ of the Poincaré group, defined on the dense domain $D$, such that the operators $U(a, \Lambda)$ are "unitary" with respect to the product $\langle\cdot, \cdot\rangle$, i.e. $\langle U \Psi, U \Phi\rangle=\langle\Psi, \Phi\rangle \Psi, \Phi \in D$, and the fields $\phi_{\alpha}$ transform covariantly under $U(a, \Lambda)$.

C.Physical States. There is a distinguished (non-trivial and maximal) subspace $\mathscr{H}^{\prime}(\mathscr{H}$, such that

c1) $\langle\Psi, \Psi\rangle \geqq 0 \quad \forall \Psi \in \mathscr{H}^{\prime}$

c2) there is a common dense domain $D^{\prime} \in \mathscr{H}^{\prime}$ such that $U(a, \Lambda) D^{\prime} \subset D^{\prime}$;

c3) the unique translationally invariant state $\Psi_{0}$ in $\mathscr{H}$, (i.e. such that $U(a, 1) \Psi_{0}$ $=\Psi_{0}$ ), called the vacuum state, is a cyclic vector with respect to the local field algebra $\mathscr{F}$, and it belongs to $D^{\prime}$.

D. Spectral Condition. The Fourier transform $\tilde{W}\left(q_{1}, \ldots, q_{n-1}\right)$ of the vacuum expectation values $\mathscr{W}\left(x_{1}, \ldots x_{n}\right)=W\left(x_{1}-x_{2}, \ldots x_{n-1}-x_{n}\right)$ of the fields $\phi_{\alpha}$ satisfy

$$
\tilde{W}\left(q_{1}, \ldots q_{n-1}\right)=0 \text { if } q_{j} \notin \bar{V}_{+} .
$$

E. Gauge Transformations. There exists a (non-trivial) group of local automorphisms $\alpha_{A}$ of $\mathscr{F}$, depending on real $C^{\infty}$ vector-valued functions $\Lambda$ with components $\Lambda_{a} \in 0_{M}$, 
$a=1, \ldots n$, (i.e. of at most slow increase), such that the infinitesimal action of $\alpha_{\Lambda}$ on the fields $\phi_{\alpha}$

$$
\phi_{\alpha}(f) \rightarrow \phi_{\alpha}(f)+\delta^{\Lambda} \phi_{\alpha}(f)
$$

is generated by local operators $J_{\mu}^{\Lambda}(x)$ in the following sense:

$$
\begin{aligned}
-i \delta^{\Lambda} \phi_{\alpha}(f) & =\lim _{R \rightarrow \infty}\left[Q_{R}^{\Lambda}, \phi_{\alpha}(f)\right] \\
& \equiv \lim _{R \rightarrow \infty} \int f_{R}(|\boldsymbol{x}|) f_{d}\left(x_{0}\right)\left[J_{0}^{\Lambda}(x), \phi_{\alpha}(f)\right] d^{4} x
\end{aligned}
$$

$\left(f_{R} \in \mathscr{D}\left(\mathbb{R}^{3}\right), f_{R}(|\boldsymbol{x}|)=1\right.$ for $|x|<R, f_{R}(|\boldsymbol{x}|)=0$ for $\left.|\boldsymbol{x}|>R+\varepsilon, f_{d} \in \mathscr{D}(\mathbb{R}), \int f_{d}\left(x_{0}\right) d x_{0}=1\right)$ $\delta^{\Lambda} \phi_{\alpha}$ depends linearly and continuously (in the $0_{M}$ topology) on $\Lambda$, and $\partial^{\mu} J_{\mu}^{\Lambda}(x)=0$. The automorphisms $\alpha_{\Lambda}$ are called local gauge transformations. The elements $\alpha_{\Lambda}$ corresponding to non-constant $\Lambda$ are called gauge transformations of the second kind.

The elements $\alpha_{\Lambda}$ corresponding to $\Lambda \equiv\left(\Lambda_{a}=\right.$ const, $\left.\Lambda_{b}=0 b \neq a\right)$ and such that there is at least one gauge transformation of the second kind with $\Lambda \equiv\left(\Lambda_{a} \neq\right.$ const, $\left.\Lambda_{b}=0 b \neq a\right)$ define the subgroup $G$ of local gauge transformations of the first kind. The corresponding local generators are denoted by $J_{u}^{a}(x)$. They are assumed to be local, translationally covariant conserved currents, with the property that there is a skew symmetric local field $G_{a}^{\mu \nu}=-G_{a}^{v \mu}$ such that

$$
\begin{aligned}
& \left\langle\Psi, J_{\mu}^{a} \Phi\right\rangle=\left\langle\Psi, \partial_{v} G_{a}^{v \mu} \Phi\right\rangle, \quad \forall \Psi, \Phi \in D^{\prime}, \\
& \mathscr{A}_{\mu}^{a}(f) D^{\prime} \subset D^{\prime}, \quad \mathscr{A}_{\mu}^{a}(f) \equiv J_{\mu}^{a}(f)-\partial^{v} G_{v \mu}^{a}(f) .
\end{aligned}
$$

The above properties are a slight adaptation of the similar well-known properties which characterize a local and covariant formulation (or gauge) of quantum electrodynamics (QED) [12]. For a familiar and simple interpretation of the above concepts we refer to the Gupta-Bleuler formulation of free QED $[12,13]$. The following remarks are meant to provide general motivations and discussion.

Remark. Property $A$ is just the standard definition of local fields. The requirement that the algebra $\mathscr{F}$ is large enough to allow approximation of every vector in $\mathscr{H}$ by vectors $\in D_{0} \equiv\left\{\mathscr{F} \Psi_{0}\right\}$ (cyclicity of the vacuum, assumption c3) can be regarded as a characteristic feature of what one should mean by a local "gauge". The only nonstandard element in $\mathrm{A}$ is the allowed possibility that the physically interesting matrix elements are computed by using a product $\langle\cdot, \cdot\rangle$ which is not the natural scalar product in $\mathscr{H}(\eta=1$ would correspond to the standard case).

Property $B$ is essentially the standard definition of covariance in QFT. The main difference is that no commitment is made about $\eta=1$ or $\eta \neq 1$. Since the covariance of the Wightman functions only requires the "unitarity" of $U(a, \Lambda)$ with respect to the product $\langle\cdot, \cdot\rangle$, in general the operators $U(a, \Lambda)$ need not be unitary and/or bounded operators.

In order to have a physically acceptable interpretation of the theory in the general case $\eta \neq 1$ one has to specify which vectors of $\mathscr{H}$ describe physical states (the correspondence need not be one-to one). This is essentially the reason for Condition $C$. 
Since the matrix elements between two "physical" states $\Phi, \Psi \in \mathscr{H}^{\prime}$ do not change if one adds to $\Phi$ and/or to $\Psi$ elements $\chi \in \mathscr{H}^{\prime}$ with vanishing " $\eta$-norm", i.e. $\langle\chi, \chi\rangle=0$, it is more convenient to characterize the physical state corresponding to $\Phi$ by the equivalence class $[\Phi]$. The quotient space $\mathscr{H}_{\text {phys }}=\overline{\mathscr{H}^{\prime} / \mathscr{H}^{\prime \prime}}, \mathscr{H}^{\prime \prime} \equiv\left\{\chi \in \mathscr{H}^{\prime}\right.$, $\langle\chi, \chi\rangle=0\}$, will be called the space of physical states (without quotation marks). $\mathscr{H}_{\text {phys }}$ is equipped with the positive definite scalar product $([\Phi],[\Psi])_{\mathscr{H}_{\text {phys }}} \equiv\langle\Phi, \Psi\rangle$ induced by $\mathscr{H}^{\prime}$.

The assumption of the uniqueness of the vacuum is essentially the statement that a physical theory corresponds to a "pure phase". The physical motivations for this have been discussed in the literature [14] and we do not insist on this point, the essential argument being that the presence of more than one vacuum state would give rise to a decomposition of the Hilbert space into phases, which describe in general different physical theories.

The spectral Condition $D$ is equivalent to

$$
\int d^{4} a e^{i p a}\langle\Psi, U(a) \Phi\rangle=0, \text { if } p \notin \bar{V}_{+},
$$

for any $\Psi, \Phi \in D_{0} \equiv\left\{\mathscr{F} \Psi_{0}\right\}$. The physical interpretation of the theory requires only a weak form of the spectral condition, namely

$\int e^{i p a} d^{4} a\langle\Psi, U(a) \Phi\rangle=0$, if $p \notin \bar{V}_{+}$,

for any $\Psi, \Phi \in D^{\prime} \subset \mathscr{H}^{\prime}$. The stronger form (2.5) is suggested by perturbation theory and it can be justified by arguments based on the asymptotic condition, the "support of the spectrum" of $U(a)$ being then dictated by the free in/out fields and in all the free field gauges known to us Equation (2.1) being satisfied.

Furthermore, the above spectral condition is strictly connected to the possibility of formulating the theory in terms of the Schwinger function (Euclidean formulation) by analytic continuation.

Property $E$ provides a precise definition of local gauge transformations. The term local here is meant to indicate a symmetry at the level of local fields, i.e. a local automorphism of the algebra of local fields (in the Lagrangian field theory language such are the local transformations of the fields which leave the Lagrangian or the equations of motion invariant). Local symmetry has to be contrasted to global symmetry (or global automorphism) which is realized when the local symmetry generates a symmetry of the states (one briefly characterizes the latter case by saying that the local symmetry is not spontaneously broken). Gauge transformations depending on the spacetime points $(\Lambda \neq$ const $)$ and the corresponding subgroup of constant phase gauge transformations are distinguished by calling them of the second and of the first kind, respectively. (Some authors use the terms local and global for this purpose.)

The group $G$ is by definition a local internal symmetry group of the theory; it has however a very special property with respect to other local internal symmetry groups that the theory may have, namely that of being associated to non-trivial gauge transformations of the second kind. In the Lagrangian quantum field theory language one would say that the equations of motion are not only invariant under the following transformation of the "charged" fields

$$
\psi(f) \rightarrow \psi(f)+i \Lambda_{a} Q^{a} \psi(f)
$$


$\Lambda_{a}=$ const, $Q^{a}$ being a finite dimensional representation of $G$, but also under the corresponding transformations of the second kind e.g.

$$
\begin{gathered}
\psi(f) \rightarrow \psi(f)+i Q^{a} \psi\left(\Lambda_{a} f\right) \\
A_{\mu}(f) \rightarrow A_{\mu}(f)+\left(\partial_{\mu} \Lambda\right)(f)
\end{gathered}
$$

for suitable non-constant functions $\Lambda \equiv\left(\Lambda_{a}=\Lambda_{a}(x)\right)$. The property of being associated in the above sense to local gauge transformations of the second kind, gives a very special character to $G$. It is interesting to note that the presently available experimental and theoretical evidence indicates that perhaps all the (local) internal symmetry groups which are relevant to elementary particle physics are of this type. This suggests that perhaps the most interesting feature of GQFT is that they offer the possibility of such a non-trivial combination of internal and space-time groups. Furthermore, the relation between the internal symmetry group $G$ and the space-time dependent gauge transformations of the second kind is of the same type as the relation between the Lorentz transformations and the space-time dependent coordinate transformations of general relativity.

Conditions (2.3) and (2.4) require some comment. In describing local automorphisms of the field algebra in axiomatic quantum field theory one has to abstract their basic properties, e.g. from Lagrangian QFT, so that one may give a characterization which does not rely on the equations of motions and/or the Lagrangian function. In the case of a continuous Lie group of local automorphisms the basic feature is provided by Noether theorem, according to which each generator $Q^{a}$ of the group is not only a constant of motion but also the space integral of the fourth component of a (local) conserved current $J_{\mu}^{a}(x)$ :

$$
Q^{a}=\int d^{3} x J_{0}^{a}(x), \quad \partial^{\mu} J_{\mu}^{a}(x)=0 .
$$

The above equations are the basic properties which can be easily translated into QFT as a characterization of a continuous local group of automorphism of the algebra of local fields, through its infinitesimal action

$$
\begin{aligned}
& \delta^{a} \phi_{\alpha}(f)=\lim _{R \rightarrow \infty}\left[Q_{R}^{a}, \phi_{\alpha}(f)\right]=\lim _{R \rightarrow \infty}\left[J_{0}^{a}\left(f_{R} f_{d}\right), \phi_{\alpha}(f)\right] \\
& \partial^{\mu} J_{\mu}^{a}(x)=0 .
\end{aligned}
$$

Equations (2.8') play a crucial role in understanding local symmetries in QFT and their spontaneous breaking. It is important to remark that the current conservation provides a local version of "charge conservation" which still retains a meaning even if a global conservation law fails to exist (symmetry breaking).

In passing from continuous (finite dimensional) Lie groups to continuous infinite dimensional (or gauge) Lie groups, the invariance of the Lagrangian or of the equations of motion yield an additional characterization. Clearly the $\Lambda=$ const subgroup $G$ of the gauge group is a finite dimensional continuous group, so that again Noether theorem applies, but now because of the connection with an infinite dimensional (or gauge) Lie group the currents $J_{\mu}^{a}(x)$ associated with its local generators $Q_{R}^{a}$ have a very special property : they can be written as the divergence of antisymmetric tensors $G_{\mu \nu}^{a}, J_{\mu}^{a}=\partial^{v} G_{v \mu}^{a}$. Condition (3) is the QFT translation of this basic feature and we take it as a characterization of those local internal symmetries 
which are associated with local gauge groups of the second kind [15]. The deep physical consequences of Condition (3) will be discussed in Section 4.

Conditions (3) and (4) can also be regarded as a kind of gauge invariance of the matrix elements between "physical" states. In fact, putting $\mathscr{A}_{\mu}^{a} \equiv J_{\mu}^{a}-\partial^{v} G_{v \mu}^{a}$, by Equation (2.3) one has $\mathscr{A}_{\mu}^{a} \mathscr{H}^{\prime} \subset \mathscr{H}^{\prime \prime}$ and therefore Condition (3) can be read as the statement that the physical states are equivalence classes with respect to the gaugetype transformations $\Psi \rightarrow \Psi+\mathscr{A}_{\mu}^{a} \mathscr{H}^{\prime}, \Psi \in \mathscr{H}^{\prime}$. It is worthwhile to stress that the existence of local gauge automorphisms (of the second kind) is strictly related to the existence of the unphysical field $\mathscr{A}_{\mu}$, which has vanishing expectation values between "physical" states, but it has a non-trivial action on local fields (gauge transformations).

In Condition A the possibility was left open that in some cases one might choose $\eta=1$ or at least positive. This would imply that all the standard Wightman axioms are satisfied and one would have a standard QFT. As we will see the existence of a non-trivial internal symmetry group $G$ associated to local gauge transformations of the second kind in the sense of property $\mathrm{E}$ (i.e. a non-trivial combination of internal and space-time groups) implies that $\eta$ must be indefinite. Thus a characteristic feature of local gauge quantum field theories is the lack of positivity.

Theorem. In a local gauge quantum field theory, if the local (internal) symmetry group $G$ associated with local gauge transformations of the second kind (Property E) has a non-trivial representation on $\mathscr{F}$, i.e. the charges $Q_{R}^{a}$ do not induce the trivial automorphism on $\mathscr{F}$, then the form $\langle\cdot, \cdot\rangle$ must be indefinite.

Proof. Consider the state $\Phi_{f} \equiv\left(J_{\mu}^{a}(f)-\partial^{v} G_{v \mu}^{a}(f)\right) \Psi_{0}$. Since $\Psi_{0} \in D^{\prime}$ (by c3), so does $\Phi_{f}$ as a consequence of Condition (4). Hence, by Condition (3)

$$
\left\langle\Phi_{f}, \Phi_{f}\right\rangle=0 \quad \forall f \in \mathscr{D} .
$$

If $\eta$ would be semidefinite ( $\geqq 0$ ), the above Equation (2.9) would imply

$$
\left\langle\Psi, \Phi_{f}\right\rangle=0 \quad \forall \Psi \in \mathscr{H}
$$

as a consequence of a generalized Schwarz' inequality, and then

$$
\Phi_{f}=0 \quad \forall f \in \mathscr{D}
$$

since $\eta$ is not degenerate.

One can show that in a local (and covariant) gauge quantum field theory a generalized version of the Reeh-Schlieder theorem holds [16], so that Equation (2.10) yields

$$
J_{\mu}^{a}=\partial^{v} G_{v \mu}^{a},
$$

and therefore

$$
\lim _{R \rightarrow \infty}\left[Q_{R}^{a}, \phi_{\alpha}(f)\right]=0,
$$

for any local field $\phi_{\alpha}(f)$, contrary to the assumption that $G$ has a non-trivial representation on $\mathscr{F}$. Thus $\eta$ cannot be semidefinite. 
In many of the formulations of GQFT discussed in the literature, the emphasis is on the property of positivity at the price of losing locality and (manifest) covariance of the basic fields (non-local and non-covariant gauges). The above theorem says that this is a general fact, i.e. gauge quantum field theories are non-standard QFT's.

The simplest candidate of a local gauge quantum field theory is provided by a local formulation of quantum electrodynamics. In this case the gauge transformations of the second kind, corresponding to the $c$-number functions $\Lambda$ satisfying $\square \Lambda=0$, are generated by the local currents

$$
J_{\mu}^{\Lambda}(x)=-\Lambda(x) \overleftrightarrow{\partial}_{\mu} \partial^{\varrho} A_{\varrho}(x)+\alpha \partial^{v}\left(\Lambda(x) F_{\mu \nu}(x)\right)
$$

$\chi$ being an arbitrary constant, and $A_{\varrho}$ denoting the vector potential.

Definition 1. The Wightman functions $W\left(q_{1} \ldots q_{n}\right)$ are said to have a "mass gap" or, equivalently, to satisfy a strong spectral condition if there is a positive $\mu$ such that

$$
W^{T}\left(q_{1} \ldots q_{n}\right)=0 \quad \forall q_{j}^{2}<\mu^{2} .
$$

The Wightman functions are said to have no mass gap if there exist at least one Wightman function $W\left(q_{1} \ldots q_{n}\right)$ for which there is no positive $\mu$ such that $W^{T}\left(q_{1} \ldots q_{n}\right)=0, \forall q_{j}^{2}<\mu^{2}$.

Remark. Since we are working in an indefinite metric theory the absence of a mass gap for the Wightman functions does not imply the absence of a mass gap in the physical spectrum, i.e. in the spectrum of $U(a)$ in $\mathscr{H}^{\prime}$.

Definition 2. The physical spectrum of $U(a)$ is defined as the union of the supports of the $\mathscr{S}^{\prime}$ distributions

$$
\begin{aligned}
& \int e^{i p a}\langle\Psi, U(a) \Phi\rangle d a \equiv U_{\Psi \Phi}(p) \\
& \Psi \in \mathscr{H}^{\prime}, \quad \Phi \in D^{\prime} .
\end{aligned}
$$

Clearly, by the spectral condition $U_{\Psi \Phi}(p)=0$ if $p \notin \bar{V}^{+}$. We will say that the physical spectrum of $U(a)$ has a mass gap $(o, \mu)$ if $\exists$ a positive $\mu$ such that $\forall \Psi \in \mathscr{H}^{\prime}, \Phi \in D^{\prime}$

$$
U_{\Psi \Phi}(p)=0 \quad \forall p \neq 0, \quad p^{2}<\mu^{2} .
$$

Otherwise the physical spectrum of $U(a)$ is said to have no mass gap.

Example. 2-dim QED where in the local Gupta-Bleuler gauge the Wightman functions have no mass gap, whereas the physical spectrum has a mass gap.

\section{Spontaneous Symmetry Breaking in Indefinite Metric QFT's}

Since we are dealing with indefinite metric quantum field theories the characterization of a spontaneously broken internal symmetry requires some careful adaptation of the standard case.

Definition 3. Let $\alpha$ denote a local internal automorphism of the field algebra $\mathscr{F}$, we will say that $\alpha$ does not generate a global symmetry or that $\alpha$ is spontaneously broken if there is at least one Wightman function which is not invariant under $\alpha$ :

$$
\left\langle\Psi_{0}, \alpha\left(\phi_{1}\right) \ldots \alpha\left(\phi_{n}\right) \Psi_{0}\right\rangle \neq\left\langle\Psi_{0}, \phi_{1} \ldots \phi_{n} \Psi_{0}\right\rangle
$$


Otherwise we will say that $\alpha$ is "unitarily" implementable and the mapping $\alpha: \phi \rightarrow \alpha(\phi)$ defines a symmetry of the theory [17].

Definition 4. A weakly continuous group of local automorphisms $\alpha_{\lambda}, \lambda \in \mathbb{R}$, is said to be generated by a local conserved current $J_{\mu}$, if $J_{\mu}(x)$ is a local Wightman field with $\partial^{\mu} J_{\mu}(x)=0$ and the variations $\phi_{\alpha} \rightarrow \phi_{\alpha}+\delta \phi_{\alpha}$ of the fields $\phi_{\alpha} \in \mathscr{F}$ are obtained by

$$
\delta \phi_{\alpha}=i \lim _{R \rightarrow \infty}\left[Q_{R}, \phi_{\alpha}\right]
$$

where

$$
Q_{R} \equiv \int J^{0}\left(\boldsymbol{x}, x_{0}\right) f_{R}(|\boldsymbol{x}|) f_{d}\left(x_{0}\right) d^{4} x
$$

and $f_{R}, f_{d}$ are test functions of compact support satisfying

$$
\begin{aligned}
& \int f_{d}\left(x_{0}\right) d x_{0}=1, \quad f_{d}\left(x_{0}\right)=0, \quad\left|x_{0}\right|>d \\
& f_{R}(x)=1 \quad x<R, \quad f_{R}(x)=0 \quad x>R+\varepsilon .
\end{aligned}
$$

Proposition 1. A weakly continuous local group of automorphisms $\alpha_{\lambda}$ generated by the local operator $Q_{R}$ is spontaneously broken if and only if there is at least one element $A \in \mathscr{F}$ for which

$$
\lim _{R \rightarrow \infty}\left\langle\Psi_{0},\left[Q_{R}, A\right] \Psi_{0}\right\rangle \neq 0 .
$$

Proof. If all the Wightman functions are invariant, then $\forall A \in \mathscr{F}$

$$
\left\langle\Psi_{0}, \alpha(A) \Psi_{0}\right\rangle=\left\langle\Psi_{0}, A \Psi_{0}\right\rangle \text {. }
$$

This implies

$$
\left\langle\Psi_{0}, \delta A \Psi_{0}\right\rangle \equiv \lim _{R \rightarrow \infty}\left\langle\Psi_{0}, i\left[Q_{R}, A\right] \Psi_{0}\right\rangle=0
$$

and therefore Equation (3.3) implies that $\alpha_{\lambda}$ is not spontaneously broken. Conversely, if the Wightman function $\left\langle\Psi_{0}, \phi_{1} \ldots \phi_{n} \Psi_{0}\right\rangle$ is not invariant under $\alpha$, then Equation (3.3) holds with

$$
A=\phi_{1} \ldots \phi_{n} \text {. }
$$

As in the standard case (positive metric) one can easily show that the commutator (3.2) is independent of $f_{d}$ for sufficiently large $R$ as a consequence of locality and current conservation. Furthermore, as in the standard case we have

Proposition 2 [18]. If the automorphism $\alpha_{R}$ generated by $Q_{R}$ is not spontaneously broken then there exist an "hermitean" operator $Q$, with domain $D_{0}=\{$ local states, i.e. states of the form $\left.A \Psi_{0}, A \in \mathscr{F}\right\}$, such that $\forall$ local field $A$

$$
Q A \Psi_{0}=\lim _{R \rightarrow \infty}\left[Q_{R}, A\right] \Psi_{0} .
$$

In extending the standard proof of Goldstone theorem [18] to the case of indefinite metric QFT's one encounters two kinds of difficulties: i) the lack of positivity, ii) the possible non-unitarity of space-time translations $U(a)$, since in an indefinite metric QFT, translation invariance requires only that $U(a)$ be $\eta$-unitary. 
The lack of positivity can be easily overcome provided the current $j_{\mu}(x)$, which generates the local automorphism, is such that the Fourier transform of the matrix elements $\left\langle\Psi_{0}, j_{\mu}(x) A \Psi_{0}\right\rangle$ are tempered measures on $\mathbb{R}^{4}$ for any local operator $A$ $[19]^{3}$. Clearly, this is true if the operators $U(a)$ are unitary, but in general the above requirement on $\left\langle\Psi_{0}, j_{\mu}(x) A \Psi_{0}\right\rangle$ looks rather artifical. As a matter of fact, in the applications to (local) gauge QFT's we will discuss interesting examples in which this property does not hold. In particular, one may show that $U(a)$ must fail to commute with the metric operator $\eta$ in order that a confinement mechanism may act [10] and the matrix elements $\left\langle\Psi_{0}, j_{\mu}(x) A \Psi_{0}\right\rangle$ cannot be the Fourier transform of tempered measures on $\mathbb{R}^{4}$ if charge screening has to occur. One has therefore to work out the general case.

Theorem 1 (Spontaneous Symmetry Breaking in Indefinite Metric Local QFT's) ${ }^{4}$ Consider a QFT defined by a set of local fields $\phi_{\alpha}, \alpha=1, \ldots n$, such that

i) the vacuum $\Psi_{0}$ is cyclic with respect to the polynomial algebra $\mathscr{F}$ of the smeared fields $\phi_{\alpha}(f)$,

ii) the Wightman functions are translationally invariant,

iii) the Fourier transforms of the Wightman functions satisfy the following spectral condition

$$
W\left(q_{1}, \ldots q_{n}\right)=0 \quad \text { if } q_{j} \notin \bar{V}_{+} .
$$

Further, assume that there is a conserved current $j_{\mu}(x)$ which is a local and translationally covariant field. Then, if the local automorphism generated by $j_{\mu}$, in the sense of Definition 4, does not generate a global symmetry of the Wightman functions (spontaneously broken symmetry), $\delta\left(p^{2}\right)$ singularities must occur in the Fourier transform of vacuum expectation values of local operators.

Proof. By Proposition 1 we have to study the matrix element

$$
\begin{aligned}
\left\langle\left[Q_{R}, A\right]\right\rangle & \equiv\left\langle\Psi_{0},\left[Q_{R}, A\right] \Psi_{0}\right\rangle \\
& =\int f_{R}(|\boldsymbol{x}|) \alpha\left(x_{0}\right)\left\langle\Psi_{0},\left[j_{0}\left(\boldsymbol{x}, x_{0}\right), A\right] \Psi_{0}\right\rangle d^{4} x \\
& \equiv \int f_{R}(|\boldsymbol{x}|) \alpha\left(x_{0}\right) J_{0}^{A}\left(\boldsymbol{x}, x_{0}\right) d^{4} x,
\end{aligned}
$$

where $A$ is a local operator $\in \mathscr{F}(\mathcal{O})$.

First, one remarks that the limit $R \rightarrow \infty$ of the above matrix element exists and it is in fact reached for finite sufficiently large $R$, because of locality. Furthermore the above limit is independent of the test function $\alpha\left(x_{0}\right) \in \mathscr{D}$ provided $\int \alpha\left(x_{0}\right) d x_{0}=1$. In fact for any two such functions $\alpha_{1}\left(x_{0}\right), \alpha_{2}\left(x_{0}\right)$ the test function

$$
\alpha^{\prime}\left(x_{0}\right)=\int_{-\infty}^{x_{0}}\left(\alpha_{1}\left(x_{0}^{\prime}\right)-\alpha_{2}\left(x_{0}^{\prime}\right)\right) d x_{0}^{\prime}
$$

has compact support, is $C^{\infty}$ and

$$
d \alpha^{\prime}\left(x_{0}\right) / d x_{0}=\alpha_{1}\left(x_{0}\right)-\alpha_{2}\left(x_{0}\right) .
$$

3 For this reason all the treatments of spontaneous symmetry breaking in indefinite metric QFT, known to us, assume this property [20]

4 This theorem has been conjectured by several people in the past, verbally or in print [27] 
Hence by current conservation

$$
\begin{aligned}
& \int d^{4} x f_{R}(|\boldsymbol{x}|)\left(\alpha_{1}\left(x_{0}\right)-\alpha_{2}\left(x_{0}\right)\right) J_{0}^{A}\left(\boldsymbol{x}, x_{0}\right) \\
& =-\int d^{4} x f_{R}(|\boldsymbol{x}|) \alpha^{\prime}\left(x_{0}\right) \partial J_{0}^{A}\left(\boldsymbol{x}, x_{0}\right) / \partial x_{0} \\
& =-\int f_{R}(|\boldsymbol{x}|) \alpha^{\prime}\left(x_{0}\right) \boldsymbol{\nabla} \boldsymbol{J}^{A}\left(\boldsymbol{x}, x_{0}\right) d^{4} x \\
& =\int d^{4} x \boldsymbol{\nabla} f_{R}(|\boldsymbol{x}|) \alpha^{\prime}\left(x_{0}\right) \boldsymbol{J}^{A}\left(\boldsymbol{x}, x_{0}\right)
\end{aligned}
$$

and for sufficiently large $R$ the last integral vanishes since the only points which contribute to the commutator are those for which $f_{R}(|\boldsymbol{x}|)=1$. Similarly, one may show that the above matrix element is independent of $f_{R}$ for $R$ sufficiently large.

The second remark [21] is that for $R$ sufficiently large, for $A \in \mathscr{F}(\mathcal{O})$, putting $A(x) \equiv U(x) A U(x)^{-1}$ one has

$$
\begin{aligned}
\left\langle\left[Q_{R}, A\right]\right\rangle & =\left\langle\left[Q_{R}, A(x)\right]\right\rangle=\left\langle\left[Q_{R}, \int d^{4} x g(x) A(x)\right]\right\rangle \\
& \equiv\left\langle\left[Q_{R}, C\right]\right\rangle
\end{aligned}
$$

for any $g \in \mathscr{D}$ such that $\int g(x) d^{4} x=1$. Thus, one might as well consider the last commutator which is in general better behaved than the original one. In fact, by using translation invariance again, one gets

$$
J_{0}^{C}\left(\boldsymbol{x}, x_{0}\right)=\int J_{0}^{A}(x-y) g(y) d^{4} y=\left(J_{0}^{A} * g\right)(x)
$$

which implies $J_{0}^{C}\left(\boldsymbol{x}, x_{0}\right) \in 0_{M}$ (i.e. it is $C^{\infty}$ and at most slow increase) and $\tilde{J}_{0}^{C}(p) \in 0_{c}^{\prime}$ (i.e. it is a tempered distribution of strong decrease) [22].

As a third step one proves a Jost-Lehmann-Dyson (JLD) representation for $J_{0}^{C}\left(x, x_{0}\right)$. Here, since the space-time translations are not necessarily described by unitary operators (Assumption ii) only requires $U(a)$ to be "unitary" with respect to the sesquilinear form $\langle\cdot, \cdot\rangle$ in terms of which the vacuum expectation values are computed, and in general $U(a)$ may not commute with the metric operator), one cannot conclude that $J_{0}^{C}(p)$ is a measure, as in the Araki et al. proof [23] of the JLD representation. One may, however, follow the pattern discussed by Wightman [24] and one obtains

$$
\begin{aligned}
J_{0}^{C}\left(f_{R}, \alpha\right)= & \int_{0}^{\infty} d \mu^{2}\left\{\int d^{3} y \varrho_{1}\left(\mu^{2}, \boldsymbol{y}\right)\left(\int \Delta\left(\boldsymbol{x}-\boldsymbol{y}, x_{0} ; \mu^{2}\right) f_{R}(|\boldsymbol{x}|) \alpha\left(x_{0}\right) d^{4} x\right)\right. \\
& \left.+\int d^{3} y \varrho_{2}\left(\mu^{2}, \boldsymbol{y}\right)\left(\int\left(\partial / \partial x_{0}\right) \Delta\left(\boldsymbol{x}-\boldsymbol{y}, x_{0} ; \mu^{2}\right) f_{R}(|\boldsymbol{x}|) \alpha\left(x_{0}\right) d^{4} x\right)\right\},
\end{aligned}
$$

where $\varrho_{1}\left(\mu^{2}, \boldsymbol{y}\right), \varrho_{2}\left(\mu^{2}, \boldsymbol{y}\right)$ are tempered distributions in the two variables, with compact support in the variable $\boldsymbol{y}$ as a consequence of local commutativity ${ }^{5}$.

As in the standard case, one can show that $\varrho_{i}\left(\mu^{2}, y\right)$ can be split in the following form [18]

$$
\varrho_{i}\left(\mu^{2}, \boldsymbol{y}\right)=\bar{\varrho}_{i}\left(\mu^{2}\right) \delta^{3}(\boldsymbol{y})+\nabla \boldsymbol{\sigma}_{i}\left(\mu^{2}, \boldsymbol{y}\right), \quad i=1,2,
$$

where $\boldsymbol{\sigma}_{i}\left(\mu^{2}, \boldsymbol{y}\right)$ are also of compact support. One can prove that the derivatives on the $\boldsymbol{\sigma}_{i}\left(\mu^{2}, \boldsymbol{y}\right)$ can be shifted to the test functions $f_{\boldsymbol{R}}(|\boldsymbol{x}|)$ and therefore they yield

5 We do not give all the details to derive Equation (3.5), the important property being the spectral condition iii). For these and other details we refer the reader to a forthcoming paper on the general mathematical discussion of the extension of the results of standard Wightman theory to gauge field theory 
vanishing contribution for large $R$, by locality $\left[f_{R}(|\boldsymbol{x}|)=1\right.$ on the points which contribute to the integral]. We are thus led to study the following expression

$$
\begin{aligned}
J_{0}^{\prime C}\left(f_{R}, \alpha\right) \equiv & \int_{0}^{\infty} d \mu^{2}\left\{\bar{\varrho}_{1}\left(\mu^{2}\right)\left(\int \Delta\left(\boldsymbol{x}, x_{0} ; \mu^{2}\right) f_{R}(|\boldsymbol{x}|) \alpha\left(x_{0}\right) d^{4} x\right)\right. \\
& \left.+\bar{\varrho}_{2}\left(\mu^{2}\right)\left(\int\left(\partial / \partial x_{0}\right) \Delta\left(\boldsymbol{x}, x_{0} ; \mu^{2}\right) f_{R}(|\boldsymbol{x}|) \alpha\left(x_{0}\right) d^{4} x\right)\right\} .
\end{aligned}
$$

The two integrals in round brackets can be evaluated explicitly and they give

$$
\int d^{3} p \tilde{f}_{R}(\boldsymbol{p})\left(2 p_{0}\right)^{-1}\left(\tilde{\alpha}\left(p_{0}\right)-\tilde{\alpha}\left(-p_{0}\right)\right)_{p_{0}=\left(\boldsymbol{p}^{2}+\mu^{2}\right)^{1 / 2}}
$$

and

$$
\frac{1}{2} i \int d^{3} p \tilde{f}_{R}(\boldsymbol{p})\left(\tilde{\alpha}\left(p_{0}\right)+\tilde{\alpha}\left(-p_{0}\right)\right)_{p_{0}=\left(\boldsymbol{p}^{2}+\mu^{2}\right)^{1 / 2}}
$$

respectively. Since, as we have shown, $J_{0}^{C}\left(f_{R}, \alpha\right)$ and, therefore, $J_{0}^{\prime C}\left(f_{R}, \alpha\right)$ do not depend on $\alpha$, in the limit of large $R$, provided $\tilde{\alpha}(0)=1$, we can choose $\alpha$ such that $\tilde{\alpha}\left(p_{0}\right)$ $=\tilde{\alpha}\left(-p_{0}\right)^{6}$. This shows that $\varrho_{1}\left(\mu^{2}\right)$ gives a vanishing contribution in the limit of large $R$, and we have to study only the contribution due to $\bar{\varrho}_{2}\left(\mu^{2}\right)$, i.e.

$$
i \int_{0}^{\infty} d \mu^{2} \bar{\varrho}_{2}\left(\mu^{2}\right) \tilde{\alpha}\left(\sqrt{\mu^{2}}\right) \equiv \bar{\varrho}_{2}[\alpha]
$$

since in the limit of large $R$ the integral (3.7) converges to $i \tilde{\alpha}\left(\sqrt{\mu^{2}}\right)$. Moreover, since $\tilde{\alpha}\left(\sqrt{\mu^{2}}\right)=\tilde{\alpha}\left(-\sqrt{\mu^{2}}\right)$ and $\tilde{\alpha}$ is an analytic function, there exists an analytic function $\beta$ such that $\beta\left(\mu^{2}\right) \simeq \tilde{\alpha}\left(\sqrt{\mu^{2}}\right)$ and Equation (3.8) defines a functional $\in \mathscr{D}^{\prime}$ and of strong decrease which depends only on the value of $\beta$ at the origin.

Thus one must have

$$
\bar{\varrho}_{2}\left(\mu^{2}\right)=\lambda \delta\left(\mu^{2}\right)
$$

and the matrix element $\left\langle\left[Q_{R}, C\right]\right\rangle$ does not vanish for large $R$ only if $\lambda \neq 0$. This proves that the Fourier transform of $J_{0}^{C}\left(\boldsymbol{x}, x_{0}\right)$ has a $\delta\left(p^{2}\right)$ singularity if the symmetry is spontaneously broken.

The next interesting question is whether the $\delta\left(p^{2}\right)$ singularities, which necessarily accompany the spontaneous symmetry breaking in indefinite metric QFT's will or will not show up in matrix elements of physical states. In the first case we will say that symmetry breaking occurs according to the genuine Goldstone mechanism; in the second case we will say that a Higgs mechanism is acting. One learns from simple examples that no general conclusion can be drawn unless additional information is provided. A clear characterization of the two phenomena will be given in the following section.

\section{Spontaneous Symmetry Breaking in Local Gauge QFT. The Higgs Mechanism}

Theorem 2 (Higgs Phenomenon). Let $G$ be a compact Lie group associated to the $A$ $=$ const gauge transformation of a local and covariant gauge field theory (Section 2, Property E) and let $Q_{R}^{i}$ denote the corresponding local generators (Section 2, Eq. (2.2)). Then, if the automorphism generated by $Q_{R}^{i}$ is spontaneously broken, the $\delta\left(p^{2}\right)$

$6 \quad$ It suffices to take $\alpha\left(x_{0}\right)$ such that $\alpha\left(x_{0}\right)=\alpha\left(-x_{0}\right)$ 
singularities, which necessarily accompany the spontaneous symmetry breaking, do not contribute to the physical matrix elements and in particular they do not give rise to physical massless particle states.

Proof. Let us suppose that the $\delta\left(p^{2}\right)$ singularity arises as a contribution from an intermediate state $\Psi_{G}$ (Goldstone like boson), in the sense

$$
\begin{aligned}
& \lim _{R \rightarrow \infty} \int\left\langle\Psi_{0},\left[J_{0}\left(\boldsymbol{x}, x_{0}\right), A\right] \Psi_{0}\right\rangle f_{R}(|\boldsymbol{x}|) \alpha\left(x_{0}\right) d^{4} x \\
& =\lim _{R \rightarrow \infty} \int d^{4} x f_{R}(|\boldsymbol{x}|) \alpha\left(x_{0}\right)\left\{\left(\eta J_{0}\left(\boldsymbol{x}, x_{0}\right) \Psi_{0}, \Psi_{G}\right)\left(\Psi_{G}, A \Psi_{0}\right)\right. \\
& \left.\quad-\left(A^{+} \Psi_{0}, \Psi_{G}\right)\left(\Psi_{G}, \eta J_{0}\left(\boldsymbol{x}, x_{0}\right) \Psi_{0}\right)\right\} \neq 0
\end{aligned}
$$

( $A^{+}$being the $\eta$-adjoint of $A$ ). Then, we can show that $\Psi_{G}$ cannot belong to $\mathscr{H}^{\prime}$ and more precisely that its component in the subspace $\mathscr{H}^{\prime}$ cannot contribute to the above integral. In fact, putting

$$
\mathscr{A}_{\mu} \equiv J_{\mu}-\partial^{v} G_{v \mu}
$$

(where $G^{v \mu}$ is the local operator introduced in Property E) one easily shows that

$$
\mathscr{A}_{R} \equiv \mathscr{A}\left(f_{R}, \alpha\right) \equiv \int d^{4} x f_{R}(|\boldsymbol{x}|) \alpha\left(x_{0}\right) \mathscr{A}_{0}\left(\boldsymbol{x}, x_{0}\right)
$$

and $Q_{R}=J\left(f_{R}, \alpha\right)$ generate the same automorphism on the local fields, since the difference $(\partial G)_{R}$ generates the trivial automorphism [25]. Therefore for $R$ sufficiently large

$$
\left\langle\left[Q_{R}, A\right]\right\rangle=\left\langle\left[\mathscr{A}_{R}, A\right]\right\rangle
$$

and Equation (4.1) becomes

$$
\begin{aligned}
\lim _{R \rightarrow \infty}\left\langle\left[\mathscr{A}_{R}, A\right]\right\rangle= & \lim _{R \rightarrow \infty} \int d^{4} x f_{R}(|x|) \alpha\left(x_{0}\right)\left\{\left(\eta \mathscr{A}_{0}(x) \Psi_{0}, \Psi_{G}\right)\left(\Psi_{G}, A \Psi_{0}\right)\right. \\
& \left.-\left(A^{+} \Psi_{0}, \Psi_{G}\right)\left(\Psi_{G}, \eta \mathscr{A}_{0}(x) \Psi_{0}\right)\right\} \neq 0 .
\end{aligned}
$$

Hence $\Psi_{G}$ cannot belong to $\mathscr{H}^{\prime}$ otherwise ${ }^{7}$

$$
\begin{gathered}
\int d^{4} x f_{R} \alpha\left(\eta \mathscr{A}_{0} \Psi_{0}, \Psi_{G}\right)=\left\langle\mathscr{A}_{0}\left(f_{R}, \alpha\right) \Psi_{0}, \Psi_{G}\right\rangle=0, \\
\left\langle\Psi_{G}, \mathscr{A}\left(f_{R}, \alpha\right) \Psi_{0}\right\rangle=0
\end{gathered}
$$

by Property $\mathrm{E}$, and one could not have $\lim \left\langle\left[Q_{R}, A\right]\right\rangle \neq 0$. This argument also shows that only the component of $\Psi_{G}$ in $\mathscr{H}^{\prime \perp}$ can contribute to the above integral and therefore the presence of the $\delta\left(p^{2}\right)$ singularity is completely accounted for by vectors which are not in $\mathscr{H}^{\prime}$. Thus, the spontaneous breaking of the symmetry does not give rise to physical massless particles.

Remark. The above theorem clarifies the conditions under which a spontaneous symmetry breaking in (local) gauge QFT occurs through the Higgs mechanism rather than the Goldstone mechanism : namely when the generators $Q_{R}^{i}$ of the internal local symmetry group are associated with local gauge transformations (of the second kind) in the sense of Property E, Section 2. Clearly a genuine Goldstone mechanism in gauge QFT is allowed for those local internal symmetries whose generators are not associated with local gauge transformations of the second kind.

7 It is not difficult to show that the same state $\Psi_{G}$ contributes to $\left\langle\left[\mathscr{A}_{R}, A\right]\right\rangle$ and to $\left\langle\left[Q_{R}, A\right]\right\rangle$. Moreover $\eta \Psi_{G} \in \mathscr{H}^{\prime \prime}$ 
Examples: 2-dim QED and the (abelian) Higgs model

It is useful to stress that the Higgs phenomenon is often accompanied by the phenomenon of charge screening [26] or, equivalently, by the lack of commutativity [27] between the metric $\eta$ and the space-time translation operators $U(a)^{8}$. This means that $U(a)$ are not unitary operators, but only unitary with respect to the sesquilinear form $\langle\cdot, \cdot\rangle$ (as required by the translational invariance of the Wightman functions).

Proposition 3. If $\mathscr{A}_{\mu}^{i}$ generates the same local automorphism as $\lambda\left(\partial^{\mu} G_{\mu \nu}^{i}-\square A_{v}^{i}\right)\left(A_{v}^{i}\right.$ being a local vector field) with $\lambda$ an arbitrary constant, then the spontaneous breaking of the automorphism generated by $\mathscr{A}_{\mu}^{i}$ implies that the translations $U(a)$ do not commute with the metric ${ }^{9}$.

Proof. Clearly for any local operator $A$

$$
\lim _{R \rightarrow \infty}\left\langle\left[\mathscr{A}^{i}\left(f_{R} \alpha\right), A\right]\right\rangle=-\lambda \lim _{R \rightarrow \infty}\left\langle\left[\square A_{0}^{i}\left(f_{R} \alpha\right), A\right]\right\rangle
$$

and if $[U(a), \eta]=0$ the right-hand side can be written as

$$
\lim _{R \rightarrow \infty}\left\{\left\langle\Psi_{0}, \square A_{0}^{i}\left(f_{R} \alpha\right) \Psi\right\rangle\left(\Psi, A \Psi_{0}\right) \text {-crossed term }\right\},
$$

where $\Psi \in E_{0} \mathscr{H}, E_{0}=\int_{p^{2}=0} d E(p)$. Then

$$
\left\langle\Psi_{0}, \square A_{\mu}^{i}(x) \Psi\right\rangle=\square\left\langle\Psi_{0}, A_{\mu}^{i}(x) \Psi\right\rangle=0
$$

which would imply no spontaneous symmetry breaking. Thus the breaking of the automorphism generated by $\mathscr{A}_{\mu}^{i}$ can occur only if $[U(a), \eta] \neq 0$.

Remark. An example exhibiting the above features is provided by the abelian case [27] (or the abelian Higgs-Kibble model) as can be seen in a local gauge where one expects that the following equations hold

$$
\partial^{\mu} F_{\mu \nu}=\square A_{v}-\partial_{v} \partial A=J_{v}+\alpha \partial_{v} \partial A .
$$

$\alpha$ being a parameter which depends on the specific choice of the gauge. In this case $\mathscr{A}_{\mu}=\alpha \partial_{\mu} \partial A=-\alpha\left(\partial^{\mu} F_{\mu \nu}-\square A_{v}\right)$ so that the condition of Proposition 3 is clearly realized.

\section{Gauge Transformations of the Second Kind and Their Spontaneous Breaking}

The existence of local automorphisms associated to local gauge transformations of the second kind, characterizes one of the basic feature of gauge quantum field theories. It is therefore of some interest to spell out some properties of those automorphisms and in particular to investigate their spontaneous breaking.

We will consider the case in which there is a group of local automorphisms $\alpha_{A}$ labelled by a linear set $S$ of functions ${ }^{10} \Lambda \in 0_{M}$, generated by currents $J_{\mu \alpha}^{\Lambda}(x), \mu$

\footnotetext{
8 This property is strictly related to the mechanism of confinement [10]

9 This Proposition is very similar to the Lemma of Ref. [27] where $\mathscr{A}_{\mu}$ and $\partial^{v} G_{v \mu}-\square A_{\mu}$ are proportional

$10 \alpha_{\Lambda}$ need not be defined for all $\Lambda \in 0_{M}$. The set $S$ is however assumed to be invariant under translations, i.e. if $\Lambda(x) \in S$ also $\Lambda_{a}(x) \equiv \Lambda(x+a) \in S$, for any $a \in \mathbb{R}^{4}$, and closed under derivations, i.e. if $\Lambda(x) \in S$, any derivative $D^{|x|} \Lambda(x) \in S$
} 
$=0,1,2,3$ ( $\alpha$ being some other possibly tensor index) having the following properties

a) $J_{\mu}^{\Lambda}(x), \mu=0, \ldots 3$ are for any $\Lambda \in S$ operator-valued tempered distributions,

b) $\partial^{\mu} J_{\mu}^{\Lambda}(x)=0$,

c) for any local $\phi_{\alpha} \in \mathscr{F}(\mathcal{O})$ the mapping $\alpha_{\Lambda}\left(\phi_{\alpha}\right)$ is continuous [35] in $\Lambda$ in the $0_{M}$ topology and the infinitesimal transformation

$\delta^{\Lambda} \phi \equiv \lim _{R \rightarrow \infty}\left[J_{0}^{\Lambda}\left(f_{R} \alpha\right), \phi_{\alpha}\right]$

depends linearly ${ }^{11}$ on $\Lambda^{12}$,

d) $J_{\mu}^{\Lambda}(x), \mu=0, \ldots 3$ are local fields, i.e. $\forall \phi_{\alpha} \in \mathscr{F}(\mathcal{O})$

$\left[J_{\mu}^{\Lambda}(g), \phi_{\alpha}\right]=0$ if $\operatorname{supp} g \times \mathcal{O}$.

Properties $\mathrm{a}-\mathrm{d}$ are suggested by simple examples of GQFT's. Perturbation theory indicates that they also hold in the Gupta-Bleuler formulation of Quantum Electrodynamics where the gauge transformations of the second kind are generated by the local current

$$
J_{\mu}^{\Lambda}(x)=-\Lambda \overleftrightarrow{\partial_{\mu}} \partial^{v} A_{v}(x)
$$

Condition $\mathrm{b}$ could be replaced by the weaker one

$$
\lim _{R \rightarrow \infty}\left[\left(\partial^{\mu} J_{\mu}^{\Lambda}\right)\left(f_{R} \alpha\right), A\right]=0, \quad \forall A \in \mathscr{F} .
$$

Further information, which is necessary for the analysis of gauge transformations of the second kind, is the commutation relation with the space-time translations, since, in general, as it is also evident from $(5.1), J_{\mu}^{A}(x)$ will not transform covariantly under space-time translations

$$
U(a) J_{\mu}^{\Lambda}(x) U(a)^{-1} \neq J_{\mu}^{\Lambda}(x+a) .
$$

One may however separate the covariant and the non-covariant behavior of $J_{\mu}^{\Lambda}$ by introducing the "field" $\dot{j}_{\mu}^{1}(y, x)$ defined ${ }^{13}$ by

$$
\dot{j}_{\mu}^{1}(f, g)=\int d^{4} z U(z) \dot{j}_{\mu}^{1}\left(f_{-z}, g\right) U(z)^{-1},
$$

where $f_{-z}(x) \equiv f(x-z)$. Thus $\dot{f}^{1}(y, 0)$ transforms covariantly in the $y$ variable

$$
U(a) \dot{j}_{\mu}^{1}(y, 0) U(a)^{-1}=\dot{j}_{\mu}^{1}(y+a, 0) .
$$

The dependence of $j_{\mu}^{1}(y, x)$ on the $x$ variable depends on the explicit transformation law of $J_{\mu}^{\Lambda}(x)$ under space-time translations. For example, if $J_{\mu}^{\Lambda}(x)$ is of the form

$$
J_{\mu}^{\Lambda}(x)=\sum_{i=0}^{N} j_{\mu}^{i}(x) \mathscr{P}^{i}(\partial) \Lambda(x) .
$$

\footnotetext{
11 We do not require that $J_{\mu}^{A}$ itself has this property since there are examples in which this property does not hold for $J_{\mu}^{A}$, but only for the commutator

12 This implies that $J_{0}^{\Lambda=0}\left(f_{R} f_{d}\right)$ generates the trivial automorphism in the limit of large $R$ and therefore without loss of generality we can put $J^{\Lambda=0}=0$ by a redefinition of $J_{\mu}^{\Lambda}: J_{\mu}^{\Lambda} \rightarrow J_{\mu}^{\Lambda}-J_{\mu}^{\Lambda=0}$

13 In general $j_{\mu}^{1}(f, g)$ will be a bilinear form defined on $D_{0} \times D_{0}, D_{0} \equiv \mathscr{F} \Psi_{0}$. One may show that $j_{\mu}^{1}(f, g)$ is actually an operator if e.g. the space-time translations commute with the metric $\eta$
} 
$j_{\mu}^{i}(x)$ being local translationally covariant fields and $\mathscr{P}^{i}$ being a polynomial in the derivatives, one has

$$
\dot{j}_{\mu}^{1}(0, x)=\dot{j}^{1 x}(0,0), \quad \Lambda_{x}(y) \equiv \Lambda(x+y)
$$

and

$$
\begin{aligned}
& \operatorname{supp}_{x} \dot{j}_{\mu}^{A}(f, x) \subset \operatorname{supp}_{x} \Lambda(x), \\
& \operatorname{supp}_{q} \tilde{\dot{j}}_{\mu}^{1}(f, q) \subset \operatorname{supp}_{q} \tilde{\Lambda}(q) .
\end{aligned}
$$

Motivated by the above remarks, in order to simplify the discussion ${ }^{14}$, we will assume that

e) the set $S$ is invariant under translations, i.e. if $\Lambda \in S$ also $\Lambda_{a}(x) \equiv \Lambda(x+a) \in S$, and there is a field $\dot{J}_{\mu}^{A}(y, x)$ which is an operator-valued tempered distribution in the $y$ variables, and an $0_{M}$ function in the $x$-variable such that

$$
J_{\mu}^{\Lambda}(x)=\dot{J}_{\mu}^{\Lambda}(x, x)
$$

and

$$
U(a) j_{\mu}^{1}(y, x) U(a)^{-1}=\dot{j}_{\mu}^{1}(y+a, x)=\dot{j}_{\mu}^{1-a}(y+a, x+a) .
$$

The above Equations (5.2) and (5.3) imply the following commutation relation between $\alpha^{\Lambda}$ and the automorphisms $T_{a}$ describing space-time translations

$$
T_{a} \alpha^{\Lambda}=\alpha^{\Lambda} T_{a} \alpha^{\Lambda-\Lambda_{a}}, \Lambda_{a}(x) \equiv \Lambda(x+a) .
$$

Equation (5.4) is also equivalent to

$$
T_{a} \alpha^{4}=\alpha^{4-a} T_{a} \text {. }
$$

Conditions a-e isolate some characteristic property of the familiar gauge transformations

$$
\begin{aligned}
\Psi(x) & \rightarrow e^{i q \Lambda(x)} \Psi(x) \\
A_{\mu} & \rightarrow A_{\mu}(x)+\partial_{\mu} \Lambda(x),
\end{aligned}
$$

and give a precise meaning to gauge transformation of the second kind, in general ${ }^{15}$.

Theorem 3 (Generalized Goldstone Theorem). Let $\alpha^{4}$ be a local automorphism generated by a current $J_{\mu}^{A}(x)$ satisfying Conditions a-e above, and $\operatorname{supp} \tilde{j}_{\mu}^{1}(f, q) \subset \bar{V}_{+} \cap \bar{V}_{-}$. Then if $\lim _{R \rightarrow \infty}\left\langle\left[Q_{R}^{A}, A\right]\right\rangle \neq 0$, for some $A \in \mathscr{F}$, there are $\delta\left(p^{2}\right)$ singularities in the vacuum expectation value $\left\langle\Psi_{0}, J_{\mu}^{\Lambda}(f) A \Psi_{0}\right\rangle$.

Remark. This theorem is a generalization of Goldstone theorem in two respects. First the local current $J_{\mu}^{\Lambda}(x)$ generating the automorphism $\alpha^{4}$ is not assumed to transform covariantly under translations. Secondly, indefinite metric is allowed and, above all, space-time translations $U(a)$ may fail to commute with the metric operator. A similar theorem was proved by Dothan and Gal-Ezer [20] and by Ferrari [20] under the assumption that the space-time translations commute with the metric; as discussed before this restriction in general precludes the application of the theorem to gauge quantum field theories exhibiting a Higgs phenomenon.

\footnotetext{
14 The essential results of this section hold under more general conditions

15 Definitions of gauge transformations similar to the one given above occur in the literature (Ref. [20]; especially the second paper). For the differences see e.g. footnote
} 
Proof. By an argument similar to that used in the proof of Theorem 1, putting $A(y) \equiv U(y) A U(y)^{-1}$ one has that

$$
\begin{aligned}
& \lim _{R \rightarrow \infty} \int d^{4} x f_{R}(|x|) \alpha\left\langle\Psi_{0},\left[\dot{f}_{0}^{4}(x, x), A\right] \Psi_{0}\right\rangle \\
& \equiv \lim _{R \rightarrow \infty} \int d^{4} x f_{R} \alpha I_{A}^{A}(x, x)=0 .
\end{aligned}
$$

$\forall A \in \mathscr{F}$, implies

$$
\lim _{R \rightarrow \infty} \int d^{4} x f_{R}(|x|) \alpha I_{A(y)}^{\Lambda}(x, x)=0
$$

$\forall A \in \mathscr{F}$, and vice versa. On the other hand, by translational covariance of $\dot{j}_{0}^{1}(x, x)$ in the first variable, one has

$$
I_{A(y)}^{\Lambda}(x, x)=I_{A}^{\Lambda}(x-y, x)
$$

and Equation (5.6) reads

$$
\lim _{R \rightarrow \infty} \int d^{4} x f_{R}(|\boldsymbol{x}|) \alpha\left(x_{0}\right) I_{A}^{A}(x-y, x)=0
$$

or

$$
\lim _{R \rightarrow \infty} \int d^{4} x d^{4} y f_{R}(|\boldsymbol{x}|) \alpha\left(x_{0}\right) I_{A}^{\Lambda}(x-y, x) g(y)=0,
$$

where $g$ is any function $\in \mathscr{D}$ such that $\int d^{4} x g(x)=1$. One is thus lead to study the function

$$
I_{A}^{\Lambda}(x) \equiv \int I_{A}^{\Lambda}(x-y, x) g(y) d^{4} y
$$

which is better behaved than $I_{A}^{A}(x, x)$. In fact it is a function $\in 0_{M}$ as can be seen by the same argument used in the proof of Theorem 1, the dependence on the second variable in $I_{A}(x-y, x)$ being $C^{\infty}$ and of at most slow increase by assumption.

Moreover, $\operatorname{supp} \tilde{I}_{A}^{\Lambda}(p) \subset \bar{V}_{+} \cup \bar{V}_{-}$since

$$
\int \tilde{I}_{A}^{\Lambda}(p) f(p) d^{4} p=\int \tilde{I}_{A}^{\Lambda}(q, p-q) g(-q) f(p) d^{4} q d^{4} p
$$

and

$$
\operatorname{supp}_{q} \tilde{I}_{A}^{\Lambda}(q, p) \subset \bar{V}_{+} \cup \bar{V}_{-}, \operatorname{supp}_{p} \tilde{I}_{A}^{\Lambda}(q, p) \subset \bar{V}_{+} \cap \bar{V}_{-}
$$

as a consequence of the spectral condition and the hypothesis of the theorem, respectively.

One may therefore write down a JLD representation for $I_{A}^{\Lambda}(x)$ as in the proof of Theorem 1. Moreover the independence of the test function $\alpha\left(x_{0}\right)$ in the large $R$ limit is proved in the same way as in Theorem 1 , as a consequence of locality and current conservation (Conditions $b$ and $d$ ). The proof of Theorem 3 then follows along the same pattern as in Theorem 1, and it will not be repeated here.

Remark 1. One may take the point of view that a local non-internal automorphism does not generate a global symmetry or that it is spontaneously broken if there is no unitary operator $V$ such that $\alpha(A)=V A V^{-1} \forall A \in \mathscr{F}$ and $V \Psi_{0}=\Psi_{0}$. This is equivalent to the property that

$$
\left\langle\Psi_{0}, \alpha(A) \Psi_{0}\right\rangle \neq\left\langle\Psi_{0}, A \Psi_{0}\right\rangle
$$


for some $A \in \mathscr{F}$. With this definition of broken symmetry the condition

$$
\lim _{R \rightarrow \infty}\left\langle\Psi_{0},\left[Q_{R}^{A}, A\right] \Psi_{0}\right\rangle \neq 0
$$

for some $A \in \mathscr{F}$, is equivalent to the spontaneous breaking of $\alpha^{A}$ and Theorem 3 can be read as a characterization of the spontaneous breaking of gauge transformations.

Remark 2. According to Theorem 4 for each $\Lambda$ such that

$$
\lim _{R \rightarrow \infty}\left\langle\Psi_{0},\left[Q_{R}^{\Lambda}, A\right] \Psi_{0}\right\rangle \neq 0
$$

for some local operator $A$, one predicts the existence of $\delta\left(p^{2}\right)$ singularities. A natural question is whether they are all independent (or unrelated) and whether one can reduce the check of Equation (5.10) for all $\Lambda$ 's to a check for a subclass of $\Lambda$ 's.

Proposition $^{16}$. If for some value of $\beta(\beta=0,1, \ldots 3), Q_{R}^{A}$ and $Q_{R}^{\partial_{\beta} A}$ denote the local charges corresponding to local currents $J_{\mu}^{\Lambda}, J_{\mu}^{\partial_{\beta} \Lambda}$ having the Properties a-e, then for $R$ sufficiently large

or

$$
\left\langle\left[Q_{R}^{\partial_{\beta} A}, A\right]\right\rangle=\lim _{a_{\beta} \rightarrow 0} i a_{\beta}^{-1}\left\langle\left[Q_{R}^{A}, U_{a}(A)-A\right]\right\rangle, a_{v}=0 v \neq \beta
$$

$$
\left\langle\left[Q_{R}^{\partial_{\beta} \Lambda}, C\right]\right\rangle=\left\langle\left[Q_{R}^{\Lambda}, C^{\prime}\right]\right\rangle,
$$

where $A$ is any local operator, $C \equiv \int A(x) g(x) d^{4} x, g \in \mathscr{D}, C^{\prime}=\int A(x) \partial_{\beta} g(x) d^{4} x$.

Thus, if Condition (5.10) holds for $Q_{R}^{\partial_{\beta} \Lambda}$ for some local operator $C$, the same condition holds for $Q_{R}^{4}$ for the operator $C^{\prime}$ and the corresponding $\delta\left(p^{2}\right)$ singularities are obviously related.

Proof. It follows easily from Equation (5.4) when written in the infinitesimal form

$$
\left(T_{a}-1\right)\left[Q_{R}^{\Lambda}, A\right]=\left[Q_{R}^{\Lambda}, T_{a}(A)-A\right]+T_{a}\left[Q_{R}^{\Lambda-\Lambda_{a}}, A\right] .
$$

When one takes for $a$ a four vector of the form $a=\lambda n_{a}, n_{a}$ being a unit form vector in $a$ fixed direction, then the vacuum expectation value of Equation (5.11), divided by $\lambda$, has a limit as $\lambda \rightarrow 0$ and

$$
\lambda^{-1}\left\langle\left[Q_{R}^{\Lambda-\Lambda_{a}}, A\right]\right\rangle \underset{\lambda \rightarrow 0}{\longrightarrow}\left\langle\left[Q_{R}^{\partial_{n_{a}} \Lambda}, A\right]\right\rangle
$$

by Condition $\mathrm{c}$ and the assumption that space-time translations define a global symmetry of the vacuum expectation values.

\section{Unbroken Symmetries in the Higgs Phenomenon}

As discussed in the Introduction one of the most interesting features usually associated to the Higgs phenomenon is the existence of massless particles associated to the unbroken generators of a spontaneously broken symmetry group $G$ (Higgs phenomenon II). The situation is just the opposite of what is predicted by the standard Goldstone theorem (for theories satisfying positivity) according to which the broken generators have massless particles associated with them (Goldstone bosons) and no information is provided by the existence of unbroken generators.

16 This Proposition occurs also in the second paper of Ref. [20] 
Now, elementary particle physics exhibits exactly the features of the Higgs phenomenon with no massless particle corresponding to the broken generators of internal symmetries and only one massless (boson) particle, the photon, corresponding to the unbroken electric charge generator.

It seems therefore of some interest to provide a rigorous discussion of this phenomenon and to isolate the basic features which characterize it. As we will see the crucial point is that the generators of the local internal symmetry group $G$ are associated to local gauge transformations in the sense of Property E.

Theorem 4. Consider a local gauge quantum field theory as defined in Section 2, with the local automorphism $\alpha^{\Lambda}$ defined for a set of $\Lambda \in O_{M}, \Lambda \neq$ const, such that $\alpha^{\Lambda}$ is generated by a local current $J_{\mu}^{\Lambda}(x)$ satisfying Conditions a-e of Section 5.

If there are fields $\psi(f)$, with non-vanishing two point function $\left\langle\Psi_{0}, \psi(f)^{+} \psi(g) \Psi_{0}\right\rangle$, such that

$$
-i \delta^{\Lambda} \psi(f) \equiv \lim _{R \rightarrow \infty}\left[Q_{R}^{\Lambda}, \psi(f)\right]=q \psi(\Lambda f),
$$

then

$$
\lim _{R \rightarrow \infty}\left\langle\Psi_{0},\left[Q_{R}^{\Lambda}, \psi(f)^{+} \psi(g)\right] \Psi_{0}\right\rangle \neq 0
$$

and if

$$
\operatorname{supp}_{q} \tilde{\dot{j}}_{\mu}^{1}(f, q) \subset \bar{V}_{+} \cap \bar{V}_{-}
$$

some of the Wightman functions have $\delta\left(p^{2}\right)$ singularities.

Proof. We consider the following vacuum expectation value

$$
\lim _{R \rightarrow \infty}\left\langle\Psi_{0},\left[Q_{R}^{A}, \psi(f)^{+} \psi(g)\right] \Psi_{0}\right\rangle .
$$

Equation (6.1) implies

$$
\lim _{R \rightarrow \infty}\left[Q_{R}^{\Lambda}, \psi(f)^{+}\right]=-q \psi(\Lambda f)^{+}
$$

so that the above vacuum expectation value becomes

$$
\int\left\langle\Psi_{0}, \psi^{+}(x) \psi(y) \Psi_{0}\right\rangle \bar{f}(x) g(y)(-\Lambda(x)+\Lambda(y)) d^{4} x d^{4} y
$$

and the condition that it vanishes $\forall f, g \in \mathscr{D}$ can be written as

$$
(\Lambda(x)-\Lambda(x+y)) S(y)=0
$$

$\forall x, y \in \mathbb{R}^{4}$, with $S(x-y) \equiv\left\langle\Psi_{0}, \psi^{+}(x) \psi(y) \Psi_{0}\right\rangle$.

Now let $I$ be an interval $I \equiv\left(x_{0}-\delta, x_{0}+\delta\right)$ such that $\Lambda(x) \neq \Lambda\left(x^{\prime}\right) \forall x, x^{\prime} \in I, x \neq x^{\prime}$. (If such an interval would not exist $\Lambda$ would be a constant contrary to the assumption of the theorem.) Then Equation (6.2) implies

$$
S(y)=0 \quad \forall y \in(0, \delta) .
$$

By the spectral condition $S(y)$ has an analytic continuation $S(z)$ in the forward tube and therefore its vanishing on an interval of its boundary implies that $S(z)$ vanishes everywhere [5, Theorem 2-14]. Thus if $S$ is non-vanishing

$$
\lim _{R \rightarrow \infty}\left\langle\Psi_{0},\left[Q_{R}^{\Lambda}, \psi(f)^{+} \psi(g)\right] \Psi_{0}\right\rangle \neq 0
$$

We can therefore apply Theorem 3 since its hypotheses are satisfied. 
Remark 1. A very simple way of satisfying the condition $\operatorname{supp}_{q} \tilde{j}(f, q)=\{q=0\}$ is to consider gauge transformation of the second kind associated to $\Lambda=c \cdot x, c$ being a constant four vector, since in this case Equation (5.3) implies

$$
\partial_{\beta}^{x} \partial_{\alpha}^{x} \dot{j}_{0}^{\Lambda}(y, x)=j_{0}^{\partial_{\alpha} \partial_{\beta} \Lambda}(y, x)
$$

and since $\partial_{\alpha} \partial_{\beta} \Lambda=0$, without loss of generality (s. footnote 12 ) we can put $j_{0}^{\partial_{\alpha} \partial_{\beta} \Lambda}(y, x)$ $=0$, i.e. $\partial_{\beta}^{x} \partial_{\alpha}^{x} j_{0}^{1}(y, x)=0$. This yields

$$
\operatorname{supp}_{q} \tilde{\tilde{j}}_{0}^{1}(f, q)=\{q=0\} \text {. }
$$

Corollary. A local QFT may allow local gauge transformations of the second kind as local automorphism of the field algebra (in the sense discussed above, Theorem 4) only if some of the Wightman functions do not have a mass gap.

The above Corollary may be regarded as a rigorous proof of the folklore statement that gauge invariance of the second kind requires the existence of massless fields. In particular, the Corollary shows that for the extension of $D H R$ analysis [28] to the case of gauge transformations of the second kind it is essential to abandon the mass gap assumption.

Remark 2. If the condition

$$
\lim _{R \rightarrow \infty}\left\langle\Psi_{0},\left[Q_{R}^{A}, A\right] \Psi_{0}\right\rangle \neq 0
$$

for some local operator $A \in \mathscr{F}$, is interpreted as a symmetry breaking condition the above Theorem 4 shows that the gauge transformations of the second kind discussed in Theorem 4 are always spontaneously broken and therefore the local field algebra admits infinite inequivalent representations associated to the infinitely "degenerate" vacua, which may be labelled in terms of the gauge functions $\Lambda(x)$. In a certain sense this provides a rigorous proof of a mechanism suggested and discussed in detail by Callan et al. [29].

Remark 3. In the simple case in which $\Lambda(x)=c^{\mu} x_{\mu}, c^{\mu}$ being a constant four vector, by Condition (5.4) if $\alpha^{\Lambda}$ can be described by a "unitary" operator $V^{\Lambda}$, then $V^{\Lambda} \Psi_{0}$ $=\Psi_{0}$, whenever the $\Lambda=$ const gauge transformation is unitarily implementable.

Thus the condition $\lim _{R \rightarrow \infty}\left\langle\Psi_{0},\left[Q_{R}^{A=c x}, A\right] \Psi_{0}\right\rangle \neq 0$ for some local $A \in \mathscr{F}$, can be interpreted as a spontaneous symmetry breaking condition without any possible reserve [30].

An interesting question is whether the $\delta\left(p^{2}\right)$ singularities predicted by Theorem 4 show up in matrix elements of physical states and give rise to massless physical states. This question does not seem to have a general answer. According to the folklore on the Higgs phenomenon one expects that the possible appearance of such massless physical particles is related to the implementability of the $\Lambda=\mathrm{const}$ transformations and also to the abelian or non-abelian character of the full $\Lambda$ $=$ const unbroken group. In the case in which the $\Lambda=$ const unbroken group is a semisimple non-abelian group, it is believed that a confinement mechanism will be acting so that massless particles with the quantum numbers of the unbroken generators $Q^{i}$ are not expected to exist and $\left\langle\Psi, Q^{i} \Phi\right\rangle=0$ for any physical states $\Psi, \Phi$. 
Theorem 5 [26]. In a local and covariant gauge QFT, if for some ithe fields $J_{\mu}^{i}(x)$ and $G_{\mu \nu}^{i}(x)$ (Property E) are observable fields and the charge $Q^{i}$ generates a non-trivial symmetry on the physical one particle states, then the physical spectrum does not have a mass gap.

Proof. Under the above assumptions $J_{\mu}^{i}$ and $G_{\mu \nu}^{i}$ define [31] local operators $\hat{J}_{\mu}^{i}(x), \hat{G}_{\mu \nu}^{i}(x)$ on $\mathscr{H}_{\text {phys }} \equiv \overline{\mathscr{H}^{\prime} / \mathscr{H}^{\prime \prime}}$ and

$$
\hat{J}_{\mu}^{i}=\partial^{v} \hat{G}_{v \mu}^{i} \text {. }
$$

Moreover the charge $\hat{Q}^{i}$ is non-trivial on the physical states of $\mathscr{H}_{\text {phys }}$. Thus, as proved by Swieca [26], the physical spectrum has no mass gap.

The assumptions entering in the Theorem are expected to be satisfied if the unbroken symmetry group is abelian. In this case, one has also the following result

Theorem 6 [30]. Let $\alpha^{\Lambda}, \Lambda=c x+b$, define a local automorphism of the field algebra $\mathscr{F}$ for any $c^{\mu}, b$ and let $\alpha^{\Lambda}$ be generated by a current $J_{\mu}^{\Lambda}(x)$ in the sense a-e of Section 5 and such that

i) there is a local field $A_{\mu}(x)$ such that $\forall f \in \mathscr{D}\left(\mathbb{R}^{4}\right)$

$$
\delta^{\Lambda} A_{\mu}(f)=\left(\partial_{\mu} \Lambda\right)(f) \quad \text { i.e. } \quad A_{\mu}(x) \rightarrow A_{\mu}+\partial_{\mu} \Lambda
$$

ii) the Fourier transform of $\left\langle J_{\mu}^{\Lambda}(x), A(g)\right\rangle, A(g) \equiv A_{v}\left(g^{v}\right), g_{v} \in \mathscr{D}\left(\mathbb{R}^{4}\right)$ is a tempered measure in the neighborhood of $p^{2}=0$, for any $g_{v}$,

iii) the $\Lambda=$ const transformations generate a global symmetry.

Then the two point function $\left\langle F_{\mu \nu}(x) F_{\varrho \sigma}(y)\right\rangle, F_{\mu \nu}=\partial_{\mu} A_{\nu}-\partial_{v} A_{\mu}$ has $\delta\left(p^{2}\right)$ singularities. In particular if $F_{\mu \nu}$ is an observable field, those $\delta\left(p^{2}\right)$ singularities appear in the physical spectrum.

Proof. The proof follows, with some slight modification, the treatment given by Ferrari and Picasso [30].

Remark. Assumption iii is crucial for the above theorem and it shows the mechanism by which the Higgs phenomenon appears for the unbroken (gauge) symmetry.

Furthermore, the above theorem shows that in QED one must expect $\delta\left(p^{2}\right)$ singularities in the photon spectral function, i.e. the photon is not an infraparticle in the sense of Schroer [32]. This justifies the basic assumption of Buchholz treatment of asymptotic condition in the zero charge sector of QED [33].

Acknowledgements. I am very grateful to the Physics Department of Princeton University and in particular to Prof. A. S. Wightman, for the kind hospitality and financial support during the academic years 1970-71, 1971-72 when this work was done and for the kind invitation during the academic year 1976-77, when this paper was written up. I also wish to thank Prof. R. Ferrari for critical comments.

\section{References}

1. Goldstone, J.: Nuovo Cimento 19, 154 (1961);

Nambu, Y., Jona-Lasinio, G. : Phys. Rev. 122, 345 (1961)

2. Goldstone,J., Salam, A., Weinberg, S. : Phys. Rev. 127, 965 (1961) 
3. Kastler,D., Robinson,D., Swieca,J.: Commun. math. Phys. 2, 108 (1966);

Ezawa,H., Swieca,J. : Commun. math. Phys. 5, 330 (1967)

4. Higgs,P.W.: Phys. Letters 12, 132 (1964); Phys. Rev. 145, 1156 (1966);

Guralnik,G.S., Hagen,C.R., Kibble,T.W.B.: Phys. Rev. Letters 13, 585 (1964); Kibble,T.W.B.: Phys. Rev. 155, 1554 (1967);

Englert, F., Brout, R.: Phys. Rev. Letters 13, 321 (1964)

5. Streater, R.F., Wightman, A.S.: PCT, spin and statistics and all that. New York: Benjamin 1964

6. Strocchi,F.: Phys. Rev. 162, 1429 (1967);

Strocchi,F., Wightman, A.S. : J. Math. Phys. 15, 2198 (1974);

Strocchi,F.: Local and covariant gauge quantum field theories; cluster property, superselection rules, and infrared problem. Invited talk at the Workshop on Quantum Electrodynamics, Aspen, June 1976

7. For a review see Coleman,S. : Secret symmetries, Erice (1973)

8. Ferrari, R. : Nuovo Cimento 19A, 204 (1974)

9. See Coleman, S. : Lectures at the Erice Summer School (1973)

10. Strocchi,F.: Phys. Letters 62 B, 60 (1976)

11. Lowenstein,J., Swieca,J.: Ann. Phys. (N.Y.) 68, 172 (1971);

Casher, A., Kogut,J., Susskind,L.: Phys. Rev. Letters 31, 792 (1973)

12. Strocchi,F., Wightman, A.S.: J. Math. Phys. 15, 2198 (1974)

13. Gupta, S. : Proc. Phys. Soc. Lond. A63, 681 (1950);

Bleuler, K. : Helv. Phys. Acta 23, 567 (1950)

14. See e.g. Wightman, A.S.: Physics Today 22, 53 (1969)

15. Strocchi,F.: Gauge groups in local field theory and superselection rules. In : Proceedings of the 4th International Colloquium on Group Theoretical Methods in Physics, Nijmegen June 1975. Lecture Notes in Physics, Vol. 50. Berlin-Heidelberg-New York: Springer 1976

16. Strocchi,F.: Local and covariant gauge quantum field theories; cluster property, superselection rules, and infrared problem. Invited talk at the Workshop on Quantum Electrodynamics, Aspen, June 1976

17. Bracci,L., Morchio,G., Strocchi,F.: Commun. math. Phys. 41, 289 (1975)

18. Swieca,J.: Goldstone theorem and related topics. In: Cargèse lectures in physics, Vol. 4 (ed. D. Kastler), p. 215, Section III. New York: Gordon and Breach 1970

19. This fact has been remarked by several people. See Reeh, H.: Symmetries, currents and infinitesimal generators (Lectures at the International Seminar at Haifa, Israel, August 1971). In: Statistical mechanics and field theory. (Eds. R. N. Sen and C. Weil). Israel: University Press 1972

20. See e.g. Dothan, Y., Gal-Ezer,E.: Nuovo Cimento 12 A, 465 (1972);

Ferrari, R. : Nuovo Cimento 14A, 386 (1973), etc.

21. Reeh,H.: Fortschr. Physik 16, 687 (1968)

22. Schwartz,L.: Théorie des Distributions, Chapter VII, Section 5. Paris: Hermann 1966

23. Araki,H., Hepp,K., Ruelle,D.: Helv. Phys. Acta 35, 164 (1962)

24. Wightman,A.S.: Analytic functions of several complex variables. In: Relations de dispersion et particules elémentaires. Les Houches Lectures 1960 (eds. C. De Witt and R. Omnes), Chapter VII. Paris: Hermann 1960

25. Ferrari, R., Picasso, L.E., Strocchi, F.: Commun. math. Phys. 35, 25 (1974)

26. Swieca,J. A.: Phys. Rev. D13, 312 (1976)

27. Ferrari, R.: Nuovo Cimento 19A, 204 (1974)

28. Doplicher, S., Haag, R., Roberts, J.: Commun. math. Phys. 13, 1 (1969); 15, 173 (1969); 23, 199 (1971)

29. Callan, C., Dashen, R., Gross, D.: Phys. Letters 63B, 334 (1976)

30. This case has been discussed in detail by Ferrari, R., Picasso, L. E. : Nucl. Phys. B31, 316(1971), under the assumption $[U(a), \eta]=0$, in the case of QED

31. Strocchi,F., Wightman, A.S. : Ref. [6]

32. Schroer, B.: Fortschr. Phys. 11, 1 (1963)

33. Buchholz, D.: To be published

Communicated by A. Jaffe

Received January 27, 1977 